
\title{
25 Research Soure \\ Large Electromagnetic Field Enhancement In Plasmonic Nanoellipse For Tunable Spaser Based Applications
}

Saqib Jamil ( $\nabla$ engr.saqibjamil@gmail.com )

Sarhad University of Science and Information Technology https://orcid.org/0000-0003-3319-6065 Javed lqbal

Sarhad University: Sarhad University of Science \& Information Technology

Adnan Daud Khan

university of engineering and technology peshawar

Waqas Farooq

Sarhad University: Sarhad University of Science \& Information Technology

\section{Research Article}

Keywords: Nanostructure, Ellipse, Plasmon coupling, Symmetry breaking, Spaser

Posted Date: August 20th, 2021

DOl: https://doi.org/10.21203/rs.3.rs-800840/v1

License: (9) This work is licensed under a Creative Commons Attribution 4.0 International License. Read Full License

Version of Record: A version of this preprint was published at PLOS ONE on March 17th, 2022. See the published version at https://doi.org/10.1371/journal.pone.0263630. 


\section{Abstract}

We theoretically demonstrated a class of plasmonic coupled elliptical nanostructure for achieving a spaser or a nanolaser with high intensity. The plasmonic ellipse is made up from gold film substrate. The proposed structure is then trialed for various light polarizations, moreover a simple elliptical nanostructure has been chosen primarily from which different cases have been formed by geometry alteration. The structure supports strong coupled resonance mode (i.e.localized surface plasmon (LSP)). The localized surface plasmon resonance (LSPR) of the investigated system is numerically examined using finite-element method (FEM). The calculations showed that the LSPR peaks and the local field intensity or near field enhancement (NFE) of the active nanosystem can be amplified to higher values by introducing symmetry breaking techniques in the proposed ellipse and its variants. The coupled nanostructure having different stages of wavelengths can be excited with different plasmonic resonance modes by the selection of suitable gain media. In addition, a small sized nanolaser with high tunability range can be developed using this nanostructure. The spaser phenomena is achieved at number of wavelengths by changing light polarization and structure alteration methods. Giant localized field enhancement and high LSPR values enable the proposed model to be highly appealing for sensing applications, surface enhanced Raman spectroscopy and much more.

\section{Introduction}

Lasers play an important role in physics and optics due to coherent light sourced nature. However, with increasing speed and size reduction in photonic devices, the role of traditional lasers is challenged due to diffraction limit of light that usually prevents the miniaturization of such devices less than half of its wavelength $[1,2]$. The localized hotspot formation in nanophotonics with intensive fields concentrations in plasmonic nanostructures are able to break the diffraction limit. The surface plasmon amplification by stimulated emission of radiation (SPASER) was first proposed by [3]. The plasmons behave like photons in conventional lasers with plasmonic cavity which helps spaser to break the diffraction limit $[4,5]$. The near field enhancement produced by nanostructures have a vast range of applications such as sensing [6], perfect light absorption [7], switching and nanocircuits [8], light speed manipulation and dispersion [9], cloaking and imaging [10], focusing and lasing [11], enhanced non-linear effects [12], surface-enhanced Raman scattering [13] and holograms. Coherent active plasmon based structures open paths for the expansion of SP based applications and investigation of the matter-light interactions at the nanoscale. Nanoscale field confinement and strong optical feedback are two critical factors for designing plasmonic lasers. The near field enhancement due to confinement can be realized by the excitement of plasmonic resonances in single metallic nanoparticles, dimer arrays and hybridized composite nanostructures [14]. LSPR modes can be controlled by the adjustment of nanoparticle's size and geometric configurations (shapes), while that of SPP modes are mainly controlled by the variation in the array's period or incident angle. Theory and experiments have shown that a metal nanoparticle array supports the SPP and LSPR coupling, where the 2-D array of the metallic nanoparticles help as a grating coupler to excite the SPP mode on the nearby metallic film [15]. The LSPR systems with strong near-field enhancement are useful 
for SERS and surface enhanced coherent anti-Stokes Raman scattering (CARS) [16], optical based sensors and absorption.

We investigated coupling of plasmons in a simple elliptical shaped nanostructure and its variants made up from silica and truncated gold ellipse, which can be fabricated using techniques suggested by $[17,18]$. The distinctive feature of an elliptical nanostructure is that a symmetry breaking can be simply obtained by extracting a small portion from the main ellipse and by rotating them at certain angles results in a number of sub-divisions or varieties. This cannot be obtained in with spherical symmetries or circular based nanostructures. We started our study from a single nano-ellipse and moved towards dimer, trimer, quadramer and their possible configurable arrangements. Near field enhancements are accomplished at different wavelengths by producing variations in the structure and incident polarizations. Different cases have been studied along $x-y$ polarization and the effect of incoming field on an output spectra with local field enhancement. Furthermore, the symmetry breaking has been done in two steps. First by taking a part from the full ellipse and then giving a particular angular shifts to these parts. The extinction spectrums of all the configurations are strongly dependent on the polarization of the incident light. Eventually, the optical response results of ellipse and variants is studied briefly and their outcomes are well explained. We studied the extinction spectrum, distribution of the induced surface charges, and the near field enhancements of gold-silica elliptical nanostructure and its variants.

\section{Model}

In this work, we have investigated several patterns of single nano-ellipse (NE) such as nano-elliptical dimer (NED), linear chain nano-elliptical trimer (LCNET), linear chain nano-elliptical quadramer (LCNEQ), symmertry broken nano-ellipse (SBNE), symmetry broken nano-elliptical dimer (SBNED), symmetry broken linear chain nano-elliptical trimer (SBLCNET) and symmetry broken linear chain nano-elliptical quadramer (SBLCNEQ). All these configurations have been formed from a single NE by adding an identical NE or by extracting a portion and then forming above mentioned configurations which are briefly discussed in later sections. The transformation from single NE to various sets is shown in Fig. 1. We found that such arrangements are much valuable for spectral lines and near field enhancement (NFE). Furthermore, we performed polarization based simulation in which we have extracted results for $x$-y polarizations along with geometric alterations for bringing a vast range of wavelength range along with giant NFE for spaser based applications such as lithography, switching, imaging and SERS etc. COMSOL with RF module have been utilized for finding optical properties/outcomes of the proposed nano-structure and its variants. Johnson and Christy data is used to find out dielectric constant of gold [19] with air as a surrounding medium. For simplicity the value of gain media is set as 2.42 for all the cases. The proposed model is placed in a cartesian coordinate system in which light is incident from $\mathrm{x}$ direction and y-direction respectively. The dimensions of the nano-structure are set as ' $a_{n}$ ' and ' $b_{n}$ ' where ' $n$ ' is an integer $(1,2,3,4)$ for various configurations. The NE is transformed to a phase known as symmetry breaking phase and the term used for nano-ellipse is symmetry broken nano-ellipse SBNE. From this set of design we have modelled different patterns mentioned above. 


\section{Results And Discussions}

In this section we have briefly described all the cases for which we have calculated our results and performed the simulations. For detailed understanding/analyzing the optical properties of a gold-silica elliptical nanostructure, it is necessary to study a gold-silica nanostructure. The optical outcome of an ellipsoid is investigated by using the plasmon hybridization theory [20].

\section{III(a). Optical Properties of a nano-ellipse NE}

We consider the truncated gold nano-ellipse (NE) surrounded by a gain media shown in Fig. 2a. The parameters of the NE are set as $a / b / t=50 / 30 / 25 \mathrm{~nm}$ respectively. Where $a$ is the outer semi-axis $b$ represents inner semi-axis while $t$ gives the thickness of the NE. The structure is placed along $x$-axis, its response is calculated for $x$-polarization case and $y$-polarization case. That is, the light is first incident from $x$-axis and in the second case the position of the nano-ellipse remained unchanged while the light was incident from y-axis. We calculated the extinction spectra Fig. 2b. and amplification obtained by the light interaction with the nanostructure. The extinction spectrum is affected by changing the direction of light on the NE, since for $x$-polaroid the spectrum is red-shifted with the peak occurred at $915 \mathrm{~nm}$ due to transverse dipolar mode and corroboration by the charge distribution, while for $y$-polaroid case the spectra shows a blue shift with a minor peak obtained at $606.2 \mathrm{~nm}$ since the transverse dipolar mode is weakened. The lowest energy mode corresponds to an anti-symmetric coupling between the plasmon resonance and the metallic nanostructure which in this case shown by y-polaroid case. While high peak arises from symmetric coupling between light and the NE. All of the systems studied herein were assumed to be in vacuum. Similarly amplification of both cases can be clearly seen in Fig. 2(c-d). It is clear that efficient coupling results in plasmon hybridization which in turn develops plasmonic effect due to which "hot-spots" are formed, so strong antibonding modes were formed for x-polarization case since, the light was efficiently coupled with the NE that led to an amplification factor of about 155 and for $y$ direction light is poorly coupled resulting the formation of bonding modes, hence, strong plasmonic effect does not occurred and we obtained less amplification of 18 . The local field enhancement (LFE) for $y$ polarized case was 7 times less than that of $x$-polarized case but can still be used for applications falling in this frequency range.

\section{III(b) Optical Properties of a symmetry broken nano-ellipse (SBNE)}

Metal based nanoparticles support plasmon resonance(s) whose energies are strongly dependent on the geometry of the nano-structure. The resonance tenability feature has brought a considerable experimental and theoretical research [21]. An important parameter in plasmonics is the effect of symmetry breaking and for nanostructures much smaller than the wavelength of incident light, only plasmons with finite dipole moments can be excited. Nanoshells are highly symmetric nanostructures in which symmetry breaking technique is normally introduced by displacing a core with respect to the outer shell may activates high-order mode(s) that become visible in the optical spectrum [22]. We have now considered a symmetry breaking case in which we have scratched a portion from the main ellipse by setting $a / b / t=50 / 30 / 25 \mathrm{~nm}$ respectively and this case is termed as symmetry broken nano-ellipse 
(SBNE). The SBNE is surrounded by a gain media in order to compensate against ohmic losses as shown in Figure.3a. We have shown that symmetry breaking leads to much larger field enhancement and the higher multipolar modes become dipole active through hybridization. Dark quadrupolar plasmon start coupling with superradiant antibonding dipolar mode which induces an asymmetric Fano resonance in the extinction spectra. By simply changing the polarization the incident light can be directly coupled to quadrupolar mode. This coupling interferes with the dispersive coupling between the superradiant and quadrupolar mode and alters the line shape of the Fano resonance. We have calculated the extinction cross section (ECS), for $x-y$ polarization which shows multiple peaks as shown in Fig. 3b. For $x-$ polarization highest peak occurred at $1142 \mathrm{~nm}$ and shows a red shift compared to the other two peaks occurred at $836.4 \mathrm{~nm}$ and $659.9 \mathrm{~nm}$. The peaks at the wavelengths of $836.4 \mathrm{~nm}$ and $659.9 \mathrm{~nm}$ shows low energy and are blue shifted since the alignment of dipolar modes was antisymmetric. Similarly, if we look at the extinction spectra for y-polarization in the same plot we see that the strongest peak occurred at $1367 \mathrm{~nm}$ and shows red shifting compared to x-polarization as well as for y-polarized case, but still it can be noticed that its height is much low as compared to $x$-polaroid due to antisymmetric interaction of light with the SBNE. Similarly other two peaks were appeared at $902.6 \mathrm{~nm}$ and $694.3 \mathrm{~nm}$ and were blue shifted with reference to $y$-polaroid but shows a slight red shifting with respect to x-polaroid peaks. Also, it is noticeable that symmetry breaking led to multi-wavelength operation compared to. Figure 3 (c-d) show the near field enhancements (NFE) for $x$-y polaroid SBNE case. The electric field distribution near the surface shows the typical dipolar resonance properties. The large electric field occurs for $x$-polarized case whose value goes up to 200, and for y-polarization the enhancement value was recorded to about 118 . Furthermore, amplification obtained from x-polarized case is about 1.5 times the y-polarized case as well as the full elliptical nano-structure. It can be seen that y-polarized case for SBNE configuration produced far better results as compared to NE, whose value was 18 which is 6.5 times less than the current case. Hence, symmetry breaking produced a huge near field enhancement (NFE) values along $x$-y polarization with multiple peaks.

\section{III(c) Optical Properties of a nano-elliptical dimer (NED)}

Plasmonic nano-elliptical dimer (NED) with closed spaced resonant particles is an arrangement in which two identical ellipses are brought close to each other and the arrangement is termed as nano-elliptical dimer (NED) structure. The coupling between two NE structures with a small separation can induce a strong, enhanced and deep subwavelength-confined optical near field inside the narrow gap by incident light. In Fig. 4a such arrangement is shown with an outer semi-axis $a=90 \mathrm{~nm}$ and an inner semi-axis $b=$ $60 \mathrm{~nm}$. The gap between two nano-structures was set as $s=5 \mathrm{~nm}, t=40 \mathrm{~nm}$ for both NE structures and the light was $x-y$ sparkled. We have plotted the extinction cross-section of the NED Fig. $4 b$, which exhibits a typical pattern when excited with x-polarized light (i. e. all electric field components are parallel to the long axis of dimer). A large red shifted peak can be seen at $1115 \mathrm{~nm}$ which typically represents the strong coupling of dimer with an incoming field. Another peak appears at the wavelength of $691.7 \mathrm{~nm}$ for same polarization but its magnitude is too small. A blue shifted peak shown in green color presents y-polaroid case and this shifting along with small height of the peak clearly depicts subradiant quadrupolar nature resulted due to poor interaction of electric field with the NED at $600 \mathrm{~nm}$. Figure 4c shows the formation of 
hot spot and amplification obtained along $x$-axis. The charges are uniformly distributed around the dimer but the hot spot is formed between the gap and this is maximum intensified point for which the enhancement maxima reaches above 620 which shows a very high amplification factor for the arrangement. Similarly, Fig. 4d shows the local field factor for $y$-polaroid case and the picture clearly shows the arrangement of charges around the dimer. In this arrangement no clear hot spot is visible due to subradiant modes, This shows, when light is incident from y-axis efficient coupling is not possible which results for the amplification factor of about 13 that is about 48 times smaller than the x-polaroid case.

\section{III(d) Optical Properties of a Symmetry broken Nano-Elliptical Dimer (SBNED)}

In this section, we have engineered a symmetry broken nano-elliptical dimer SBNED by setting all the structural parameters same as that of section III(b) and adding SBNE as depicted in Fig. 5a. The SBNED is an ideal candidate for analysis since it supports polarization of light at different angles, multipolar progression and dark modes whose tenability can be adjusted by incident light. SBNED instigated dark plasmonic modes to overlap with a bright modes resulting in the fortification of the plasmonic effect. The extinction spectra for $x-y$ luminance is shown in Fig. $5 b$. Five and three peaks appeared for $x$-polarization and y-polarization respectively because of the angular momentum contributions. The peak formed at the wavelength value of $1494 \mathrm{~nm}$ (blue-line) shows red shifting and represents the negative parity dipoles.

However, heights shown at $1199 \mathrm{~nm}$ and $865.4 \mathrm{~nm}$ represents strong mixing of positive parity bright modes with dark modes. The peak at the wavelength of $680.6 \mathrm{~nm}$ shows blue shifting with a strong magnitude compared to $1001 \mathrm{~nm}$ peak. Similarly, y-polarization produced a peak at $1381 \mathrm{~nm}$, shows a dipolar mixture of both NEs. Dark quadrupolar modes created low magnitude peaks at the wavelengths of $921.6 \mathrm{~nm}$ and $699.3 \mathrm{~nm}$ respectively. The tuning in these plots may be developed by alteration in the geometry or gap variation which is highly suitable biosensors [23]. Figure 5(c-d) show the NFEs for $x-y$ polarizations, in which the distribution of charges for $x$-luminance is far better than $y$-luminance. The charge distribution occurs in the gaps along with the outer surfaces of each NE. Four hot spots are visible, two are between the gap position and other two at the tips of the NE showing that huge plasmonic effect occurred at these points and charges are strongly confined and produced NFE value of 238. Similarly, y-polarization shows less charge distribution around the structure presenting weak hybridization and produced NFE of about 110 .

\section{III(e) Optical Properties of a Linear Chain Nano-Elliptical Trimer (LCNET)}

When a plasmonic nanoparticle is built from more than two nanoparticles, both the shape and energy of its plasmonic modes strongly depend on the arrangement of the individual particle. We now extend our work to three elliptical parts i.e each SBNE is placed along x-y plane with a fixed separation of $s=5 \mathrm{~nm}$ forming a linear chain nano-elliptical trimer (LCNET) configuration in which each SBNE is covered with the gain media layer, the LCNET is tailored in such a way that both semi-axis value and thickness for each SBNE remained unchanged and kept fixed at $a=50 \mathrm{~nm}$ and $b=30 \mathrm{~nm}$ and $t=25 \mathrm{~nm}$ respectively as shown in Fig. 6a. Trimer configuration offer a rich spectrum of possible outcomes for coupling between 
nanoparticles. New physical phenomena may be realized by symmetry alterations. The extension is performed to further optimize the results and bring variety of wavelength ranges for multiple applications. Figure $6 \mathrm{~b}$ shows the extinction spectrum in which strong bright dipole dipole bonding produced a peak for x-polaroid case at $902.1 \mathrm{~nm}$, while at $684.3 \mathrm{~nm}$ another peak appeared due to dark octupolar modes. A Fano resonance is visible at about $568 \mathrm{~nm}$. Similarly, for y-polaroid case a weak and low height peak occurred at $537 \mathrm{~nm}$ due to octupolar modes since for this case the light coupling with the structure was not efficient hence failed to generate antibonding modes. Figure 6(c-d) shows the distribution of charges and formation of spots for each $x-y$ polarization respectively. It can be seen that strong hot spots are formed in the gap positions in Fig. $6 \mathrm{c}$ due to efficient coupling while in Fig. $6 \mathrm{~d}$, most of the plasmons are scattered and failed to be confined between structural gaps, hence a large NFE of 930 is obtained for xpolaroid case while this value dropped to about 7 for $y$-polaroid case which is about 132 times less than $x$-luminance version. Furthermore, $x$-polaroid NFE value is much high compared to all the preceding cases while $y$-polaroid is the smallest. Similarly, the energy level of the peak is also low compared to above cases.

\section{III(f) Optical Properties of a Symmetry broken Linear Chain Nano-Elliptical Trimer (SBLCNET)}

We have now brought three symmetry broken elliptical parts i.e each SBNE is placed in $x-y$ plane having same parameters as that of section II(b), thus forming a symmetry broken linear chain nano- eliptical trimer (SBLCNET) with a fixed separation of $s=5 \mathrm{~nm}$ as shown in Fig. 7a.

Figure 7b shows the extinction spectrum of the SBLCNET in which it can be clearly seen that for each case i.e $x$-y polarization four peaks were appeared. The blue line presents $x$-polarization and it offered a wavelength spectrum in the range of $600 \mathrm{~nm}$ to $1650 \mathrm{~nm}$ approximately, with the highest rise at $1222 \mathrm{~nm}$ formed due to strong mixing of bright modes. Another broad red shifted peak is visible at $1587 \mathrm{~nm}$ which represents antibonding modes. Two blue shifted peaks raised at the wavelengths of $865.4 \mathrm{~nm}$ and 711 $\mathrm{nm}$ respectively, with the formation of Fano resonances. Similarly, SBLCNET offered multiple wavelength range with slight low heighted peaks for y-polarization at the positions of $1366 \mathrm{~nm}, 928.3 \mathrm{~nm}, 699.3 \mathrm{~nm}$ and $573.5 \mathrm{~nm}$ respectively. Hence this configuration generated a multi-wavelength spectrum range for numerous optical applications covering this area. The near field enhancement result for $x$-polarization is shown in Figure $7 \mathrm{c}$ in which the charges have captured all the gaps between each unit but the major hot spots are visible at the two tips of the SBNE-3. This occurred due the strong coupling of light and LCNET which energized the dark and bright modes through hybridization and produced a NFE of about 605 . Similarly, y-polarization case is depicted in Figure $7 d$ in which the charges are distributed between the gaps in the LCNET structure. However, a strongly confined hot spots were not generated in this case due to poor coupling of light and formation of dark modes but still a weak hot spot version at the tips of the SBNE-3 may be found which shows that the majority charges were confined at these points and led to the near field enhancement of about 128 . The NFE produced by y-polaroid luminance is far more than compared to y-polaroid of LCNET while x-polaroid NFE value of SBLCNET is less compared to LCNET, however symmetry breaking produced a vast range of wavelength spectrum which in turn introduces tenability and maybe deployed for number of applications. 


\section{III(g) Optical Properties of a Linear Chain Nano-Elliptical Quadramer (LCNEQ)}

We further extend our investigation by adding another SBNE with a previous LCNET structure, introducing three gaps between each SBNE and again the separation is kept constant at $s=5 \mathrm{~nm}$. Also the semi-axis parameters are fixed at $a=50 \mathrm{~nm}$ and $b=30 \mathrm{~nm}$ with thickness $t=25 \mathrm{~nm}$ respectively for each SBNE, the overall arrangement is termed as linear chain nano-elliptical quadramer (LCNEQ). The angle between each SBNE is kept as $\theta=0^{\circ}$ along with the gain media coating as shown in Fig. 8a. The extinction spectrum is shown in Fig. $8 \mathrm{~b}$ and is identical to Fig. $6 \mathrm{~b}$ in shape with a peak appeared at $956.2 \mathrm{~nm}$ due to bright dipole dipole bonding modes but have a high energy. Similarly dark octupolar modes activated at $684.3 \mathrm{~nm}$ and a Fano resonance achieved at $597.3 \mathrm{~nm}$. The y-polaroid curve is also identical to Fig. $6 \mathrm{~b}$ with a small peak appeared due to octupolar modes at $535 \mathrm{~nm}$. The charge distributions and hot spot formation for $\mathrm{x}-\mathrm{y}$ luminance is shown in Fig. 8(c-d) respectively with a NFE value of about 1019 and 8 for each case.

\section{III(h) Optical Properties of a Symmetry broken Linear Chain Nano-Elliptical Quadramer (SBLCNEQ)}

Here we have tailored SBLCNET by adding another SBNE to form symmetry broken linear chain nanoelliptical quadramer (SBLCNEQ) as shown in Figure 9a. This configuration has been developed for further investigation the effects of polarization. The extinction spectrum is shown in Figure $9 \mathrm{~b}$, which offers a rich wavelength range by producing five peaks for x-polaroid case with healthy heights. The peak at 1627 $\mathrm{nm}$ and $1234 \mathrm{~nm}$ is the highest peak representing the efficient coupling of light and activation dipole dipole modes. A small peak appeared at $1051 \mathrm{~nm}$ due to negative parity mode. Furthermore, a peak at $889.5 \mathrm{~nm}$ showing a good hybridization of dark and bright modes while a $719 \mathrm{~nm}$ peak represents a blue shifted peak and has a relative small height compared to other peaks. Similarly, y-polaroid case is shown by blue line with four peaks at $1366 \mathrm{~nm}, 908.5 \mathrm{~nm}, 707 \mathrm{~nm}$ and $573.5 \mathrm{~nm}$ respectively. This case also provided a good wavelength range but the peak heights are far low compared to x-polarized version mainly due to poor interaction of incident light from y-direction causing less mixing of each SBNE charges.

Figure 9 (c-d) depicts the near field enhancement (NFE) for x-y polarizations. Since charge confinement mainly occurs between the gaps and LCNEQ offered such gap ranges hence the enhancement achieved for x-polarized reaches upto 640 which is the highest enhancement in all the cases discussed above. Similarly, for y-polarization the light was poor coupled with the LCNEQ compared to $x$ direction hence the enhancement value recorded for this case is about 124 . Hence, this configuration offers a vast wavelength range but slight low NFE values for either case, compared to LCNEQ.

Finally we have summarized all the targeted parameters for $x$-y polarization that are achieved so far from this study in Table 1 and Table 2 respectively. The details listed in both tables further describes that symmetry breaking provides a variety of peaks and a vast area along the wavelength spectrum. These parameters clearly depicts the dominancy of x-polaroid cases over $y$-polaroid mainly due to light coupling effects. Furthermore, Fig. 10 and Fig. 11 shows the achieved NFE for x-y polaroid cases in which it can be clearly seen that simple structures configurations for $\mathrm{x}$-polarization shows higher values compared to $\mathrm{y}$ - 
polaroid. However, this is not the case for symmetry breaking in which high NFE values have been achieved even for $y$-polarization which shows that the conducted study is much fruitful for variety of spaser based applications.

Table 1

Characteristic parameters of the Elliptical nano-structures for the $x$ polarization with outer semiaxis $a=90 \mathrm{~nm}$ and inner semi axis $b=60 \mathrm{~nm}$, thickness $\mathrm{t}=40 \mathrm{~nm}$ for NE, NED, LCNET and LCNEQ. While fixing $a=50 \mathrm{~nm}$ and $b=30 \mathrm{~nm}$, thickness $\mathrm{t}=25 \mathrm{~nm}$ for SBNE, SBNED, SBLCNET and SBLCNEQ

\begin{tabular}{|lllllllll|}
\hline $\begin{array}{l}\text { Structure } \\
\text { Type }\end{array}$ & NE & SBNE & NED & SBNED & LCNET & SBLCNET & LCNEQ & SBLCNEQ \\
\hline $\begin{array}{l}\text { No. of } \\
\text { Peaks }\end{array}$ & 1 & 3 & 2 & 5 & 3 & 4 & 3 & 5 \\
\hline Wavelength & 915 & 659.9 & 691.7 & 680.6 & 568 & 711 & 597.3 & 719 \\
(nm) & & 836.4 & 1115 & 865.4 & 684.3 & 865.4 & 684.3 & 889.5 \\
& & 1142 & & 1001 & 902.1 & 1222 & 956.2 & 1051 \\
& & & & 1199 & & 1587 & & 1234 \\
NFE & 126 & 180 & 620 & 238 & 930 & 605 & 1019 & 640 \\
\hline
\end{tabular}

Table 2

Characteristic parameters of the Elliptical nano-structures for the y polarization with outer semi-axis $a=$ $90 \mathrm{~nm}$ and inner semi axis $b=60 \mathrm{~nm}$, thickness $\mathrm{t}=40 \mathrm{~nm}$ for NE, NED, LCNET and LCNEQ. While fixing a $=50 \mathrm{~nm}$ and $\mathrm{b}=30 \mathrm{~nm}$, thickness $\mathrm{t}=25 \mathrm{~nm}$ for SBNE, SBNED, SBLCNET and SBLCNEQ

Structure NE SBNE NED SBNED LCNET SBLCNET LCNEQ SBLCNEQ
Type

$\begin{array}{lllllllll}\text { No. of } & 1 & 3 & 1 & 3 & 1 & 4 & 1 & 4\end{array}$

Peaks

$\begin{array}{lllllllll}\text { Wavelength } & 606 & 1367 & 600 & 699.3 & 537 & 577.5699 .3 & 535 & 575 \\ & 902.6 & & & & 928.31366 & & 707 \\ (\mathrm{~nm}) & & 694.3 & & 921.6 & & & & \end{array}$

$1381 \quad 908.5$

1366

$\begin{array}{lllllllll}\text { NFE } & 18 & 118 & 13 & 110 & 7 & 128 & 8 & 124\end{array}$

*NE: Nano-Ellipse, SBNE: Symmetry Broken Nano-Ellipse, NED: Nano-Elliptical Dimer, SBNED: Symmetry Broken Nano-Elliptical Dimer, LCNET: Linear Chain Nano- Elliptical Trimer, SBLCNET: Symmetry Broken 
Linear Chain Nano- Elliptical Trimer, LCNEQ: Linear Chain Nano-Elliptical Quadramer, SBLCNEQ: Symmetry Broken Linear Chain Nano-Elliptical Quadramer

\section{Conclusion}

The generation of high order plasmon mode(s) have been investigated along with large near field enhancement (NFE) in a nano-elliptical configuration and its variants for $x$-y polarized light. Simple NE showed a uniform distribution of charges and hot spot formation with a good line shape. Same parametric values were applied to a NED configuration which offered an efficient coupling of dark quadrupolar with bright dipolar modes which developed two peaks along the spectrum. Symmetry breaking technique has been applied on NE to form a SBNE structure, since symmetry breaking is an ideal test bed for enhancement layout of applied parameters. This method brought three peaks at different wavelengths due to mixing of odd and even modes. We further performed extension to form a trimer and quadramer structures that brought a huge variety of wavelengths with formation of multiple peaks and hotspots along with high NFE values. To, conclude mono, dimer, trimer and quadramer simple and symmetry broken variants are ideal for producing well-defined resonance tunability and higher order dark plasmonic modes in the near infrared (NIR) and visible regions, along with huge NFE values which may be useful for numerous spaser based applications like switching, SERS, slow light and bio-medical.

\section{Declarations}

Author contribution: Conceptualization, Saqib Jamil and Adnan Daud Khan .; methodology, Adnan Daud Khan.; software, Saqib Jamil .; validation, Javed Iqbal., Saqib Jamil, Adnan Daud Khan. and Waqas Farooq.; formal analysis, Saqib Jamil.; investigation, Saqib Jamil. and Javed Iqbal.; resources, Saqib Jamil.; data curation, Saqib Jamil.; writing-original draft preparation, Saqib Jamil and Adnan Daud Khan.; writing-review and editing, Javed Iqbal and Waqas Farooq.; visualization, Saqib Jamil.; supervision, Adnan Daud khan.; project administration, Adnan Daud Khan.

Ethical Statement: Hereby, I Saqib Jamil, consciously assures that for the manuscript titled "Large electromagnetic field enhancement in plasmonic nanoellipse for tunable spaser based applications" the following is fulflled:

1. This material is the authors' own original work, which has not been previously published elsewhere.

2. The paper is not currently being considered for publication else where.

3. The paper refects the authors' own research and analysis in a truthful and complete manner.

4. The paper properly credits the meaningful contributions of co-authors and co-researchers.

5. The results are appropriately placed in the context of prior and existing research. 
6. All sources used are properly disclosed (correct citation). Literally copying of text must be indicated as such by using quotation marks and giving proper reference.

7. All authors have been personally and actively involved in substantial work leading to the paper, and will take public responsibility for its content.

The violation of the Ethical Statement rules may result in severe consequences.

I agree with the above statements and declare that this submission follows the policies of Plasmonics Journal as outlined in the Guide for

Authors and in the Ethical Statement.

Consent to Participate:Informed consent was obtained from all individual participants included in the study.

Consent for Publication: There are no case studies involve in the current work. Therefore, this declaration is not applicable in our case.

Conflict of Interest: The authors declare no competing interests.

Funding: No funding available

Availability of data and material: All the data is included in the manuscript

Code availability: No codes are associated with our work

\section{References}

1. Zhang, P., et al., A single-emitter gain medium for bright coherent radiation from a plasmonic nanoresonator. Acs Photonics, 2017. 4(11): p. 2738-2744.

2. Hill, M.T. and M.C. Gather, Advances in small lasers. Nature Photonics, 2014. 8(12): p. 908-918.

3. Bergman, D.J. and M.I. Stockman, Surface plasmon amplification by stimulated emission of radiation: quantum generation of coherent surface plasmons in nanosystems. Physical review letters, 2003. 90(2): p. 027402.

4. Stockman, M.I., Spasers explained. Nature Photonics, 2008. 2(6): p. 327-329.

5. Wang, D., et al., Structural engineering in plasmon nanolasers. Chemical reviews, 2017. 118(6): p. 28652881.

6. Yi, Z., et al., Nanoribbon-ring cross perfect metamaterial graphene multi-band absorber in THz range and the sensing application. Results in Physics, 2019. 14: p. 102367. 
7. Cen, C., et al., Numerical investigation of a tunable metamaterial perfect absorber consisting of twointersecting graphene nanoring arrays. Physics Letters A, 2019. 383(24): p. 3030-3035.

8. Chen, J., et al., Plasmonic EIT-like switching in bright-dark-bright plasmon resonators. Optics express, 2011. 19(7): p. 5970-5978.

9. Jia, W., et al., Dynamically tunable optical properties in graphene-based plasmon-induced transparency metamaterials. Chinese Physics B, 2019. 28(2): p. 026102.

10. Ma, Q., et al., Super-resolution imaging by metamaterial-based compressive spatial-to-spectral transformation. Nanoscale, 2017. 9(46): p. 18268-18274.

11. Liu, M., et al., Highly efficient plasmonic nanofocusing on a metallized fiber tip with internal illumination of the radial vector mode using an acousto-optic coupling approach. Nanophotonics, 2019. 8(5): p. 921-929.

12. Zhang, J., et al., An engineered CARS substrate with giant field enhancement in crisscross dimer nanostructure. SciEntific REPORtS, 2018. 8(1): p. 1-9.

13. Liu, M., et al., Plasmonic tip internally excited via an azimuthal vector beam for surface enhanced Raman spectroscopy. Photonics Research, 2019. 7(5): p. 526-531.

14. Wang, J., et al., Unmodified hot spot in hybridized nanorod dimer for extended surface-enhanced Raman scattering. Journal of Physics and Chemistry of Solids, 2020. 136: p. 109125.

15. Chu, Y., M.G. Banaee, and K.B. Crozier, Double-resonance plasmon substrates for surface-enhanced Raman scattering with enhancement at excitation and stokes frequencies. ACS nano, 2010. 4(5): p. 28042810.

16. Wang, J., et al., Theoretical investigation of a multi-resonance plasmonic substrate for enhanced coherent anti-Stokes Raman scattering. Optics express, 2017. 25(1): p. 497-507.

17. Mukherjee, S., et al., Fanoshells: nanoparticles with built-in Fano resonances. Nano letters, 2010. 10(7): p. 2694-2701.

18. Bardhan, R., et al., Nanosphere-in-a-nanoshell: a simple nanomatryushka. The Journal of Physical Chemistry C, 2010. 114(16): p. 7378-7383.

19. Johnson, P.B. and R.-W. Christy, Optical constants of the noble metals. Physical review B, 1972. 6(12): p. 4370.

20. Prodan, E., et al., A hybridization model for the plasmon response of complex nanostructures. science, 2003. 302(5644): p. 419-422. 
21. Nehl, C.L. and J.H. Hafner, Shape-dependent plasmon resonances of gold nanoparticles. Journal of Materials Chemistry, 2008. 18(21): p. 2415-2419.

22. Wu, Y. and P. Nordlander, Plasmon hybridization in nanoshells with a nonconcentric core. The Journal of chemical physics, 2006. 125(12): p. 124708.

23. Dodson, S., et al., Optimizing electromagnetic hotspots in plasmonic bowtie nanoantennae. The journal of physical chemistry letters, 2013. 4(3): p. 496-501.

\section{Figures}
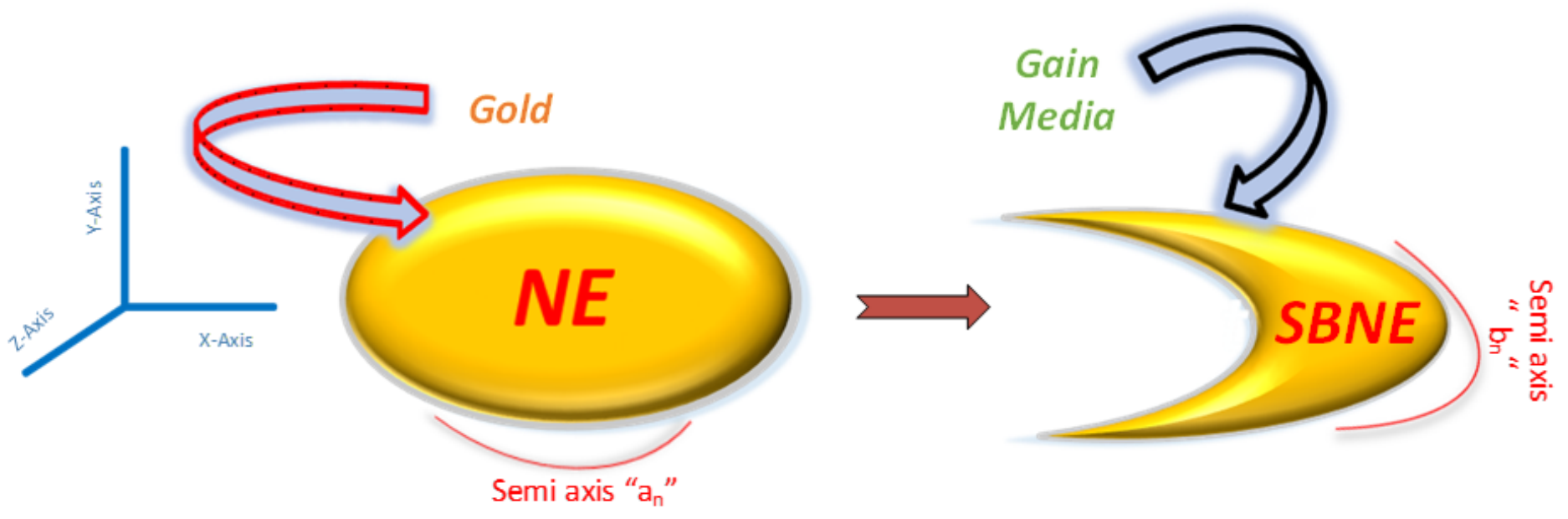

\section{Figure 1}

Transformation from single nano-ellipse (NE) to symmetry broken nano-ellipse (SBNE) 

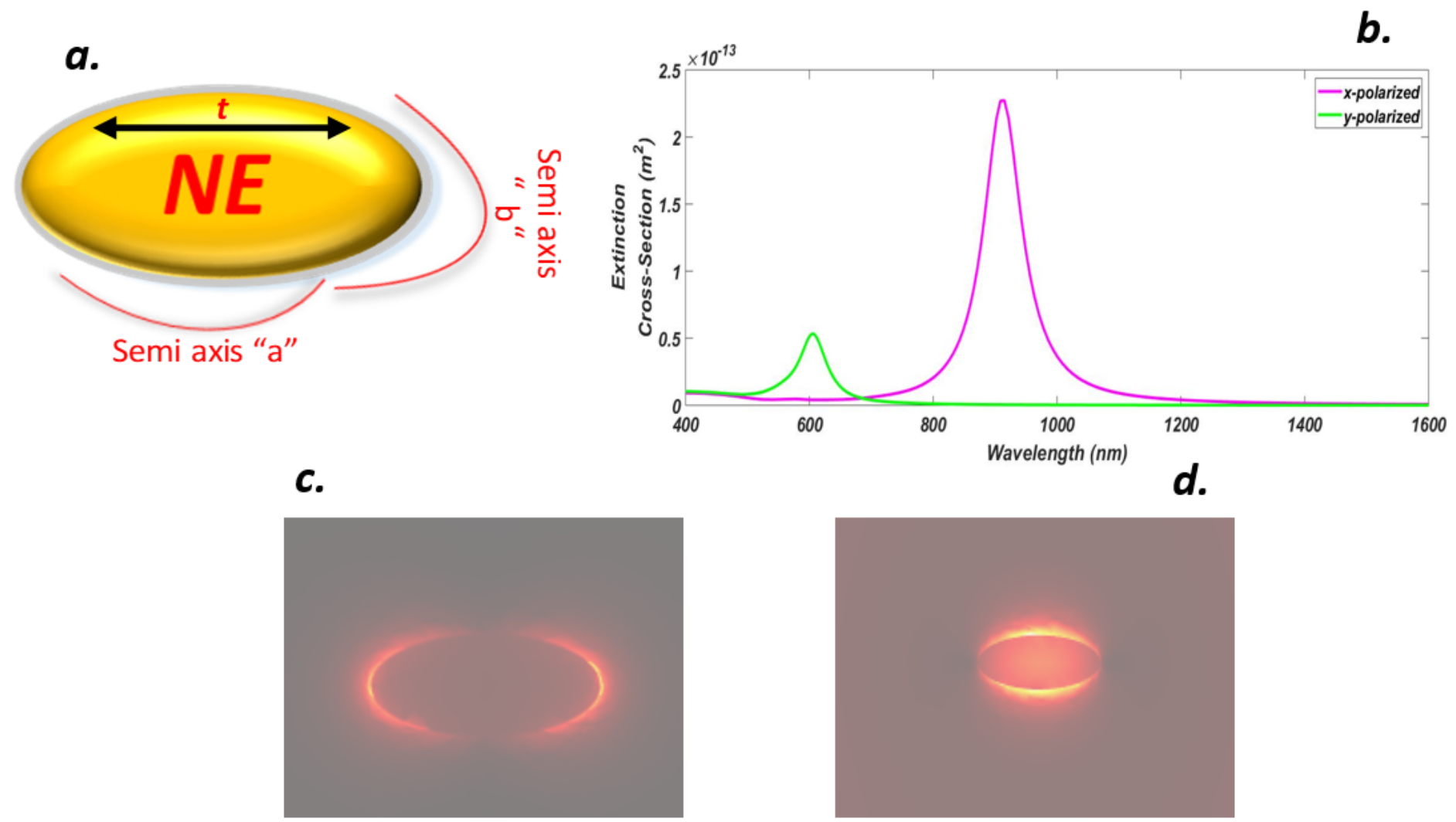

20

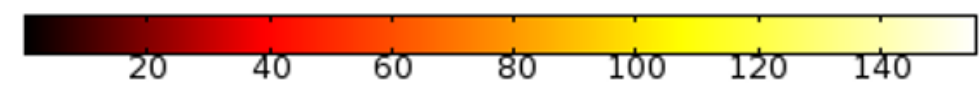

- 140

\section{Figure 2}

a.Truncated Gold Nano-ellipse (NE) with gain media coating b. ECS of NE for $x-y$ polarization c. NFE of NE for x-polarization d. NFE of NE for y-polarization 

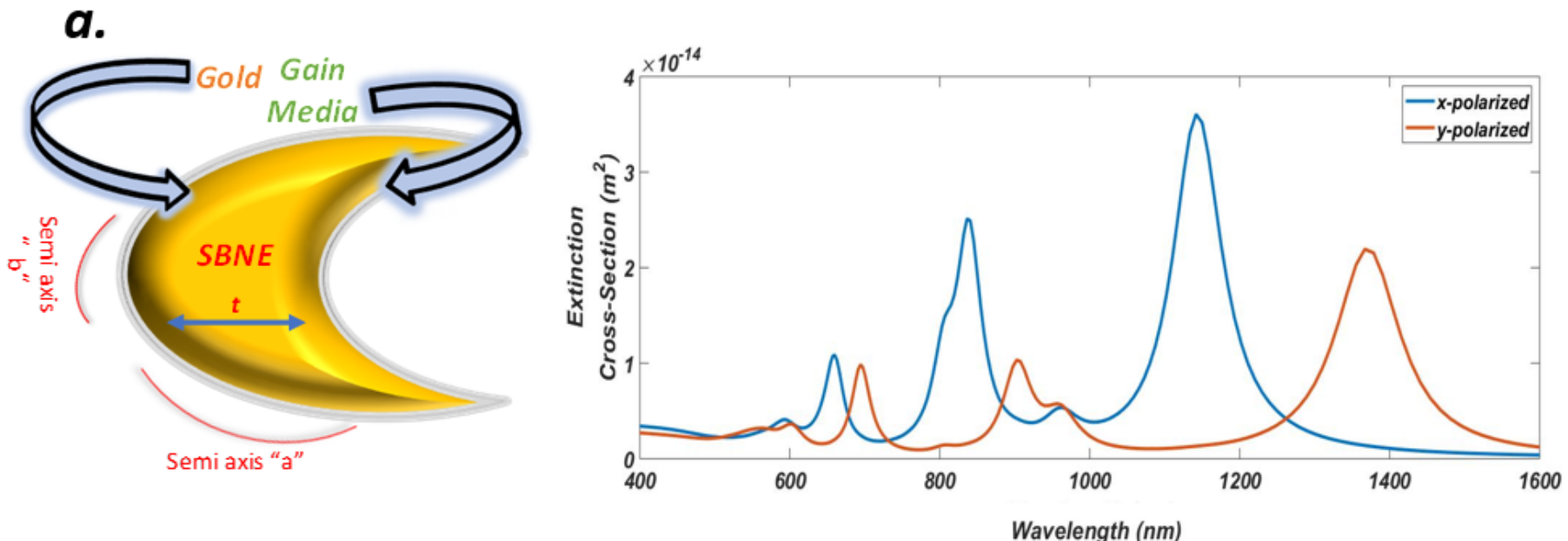

b.

C.
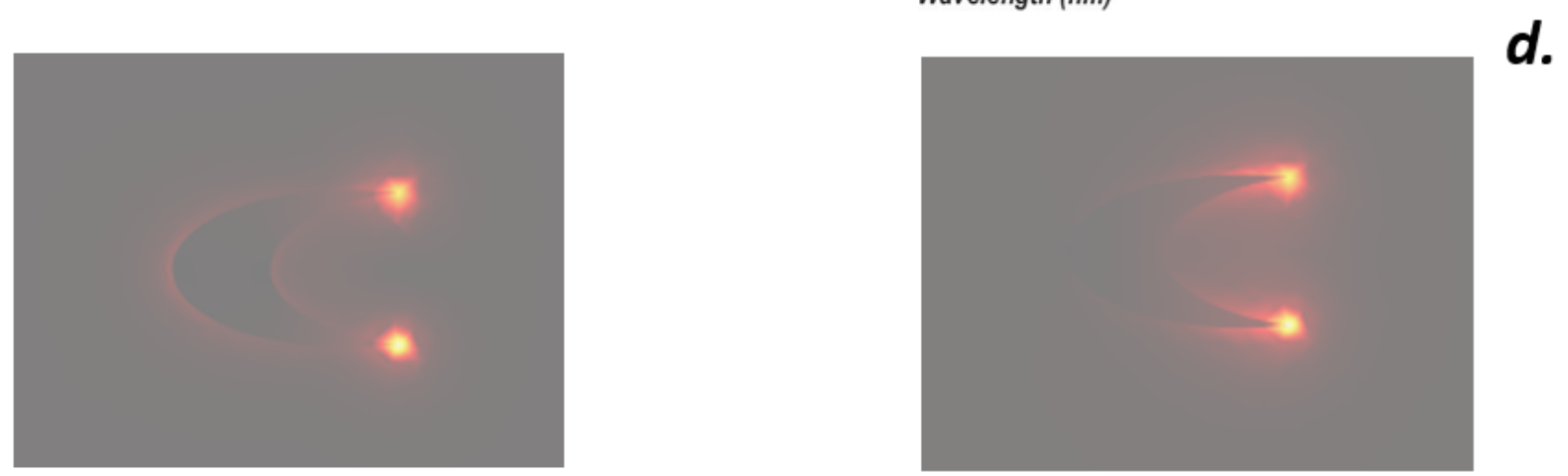

20

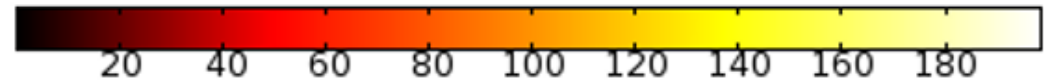

$\mathbf{\Delta} 180$

\section{Figure 3}

a. Truncated Gold Symmetry broken nano-ellipse (SBNE) with gain media coating b. ECS of SBNE for $x-y$ luminance $c$. NFE of SBNE for x-polarization d. NFE of SBNE for y-polarization 

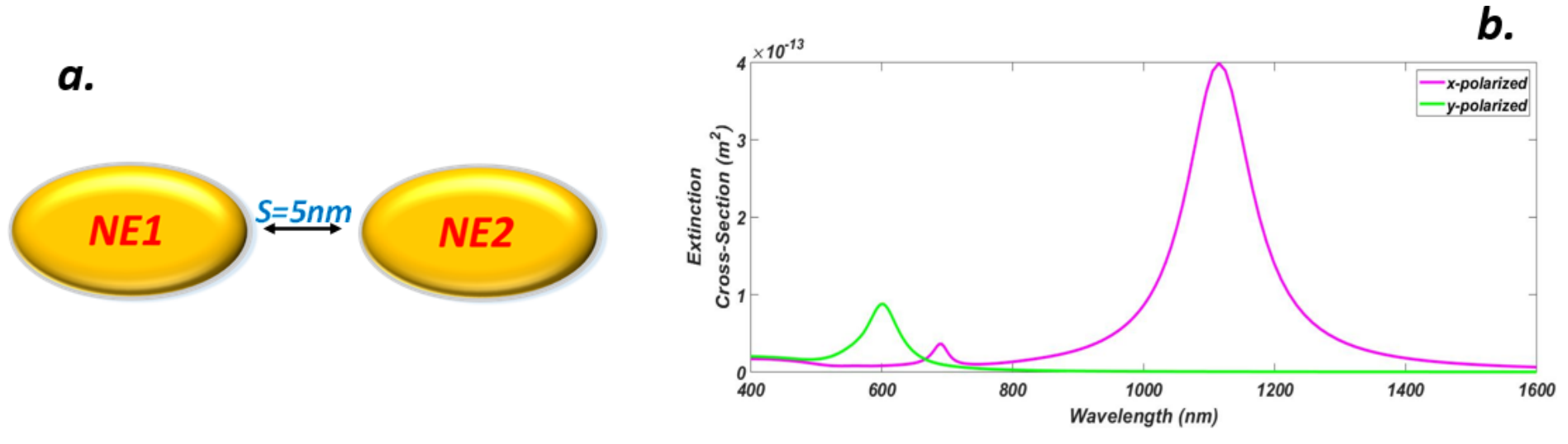

\section{c.}

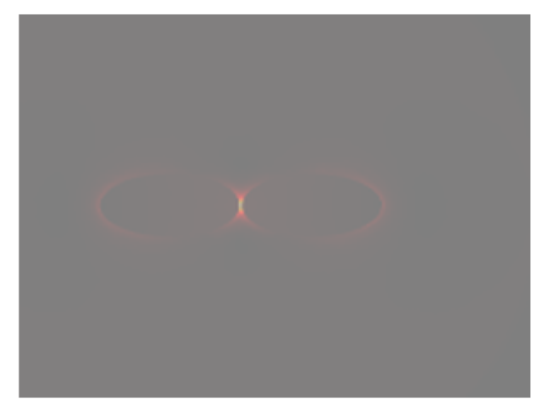

$\nabla 100$

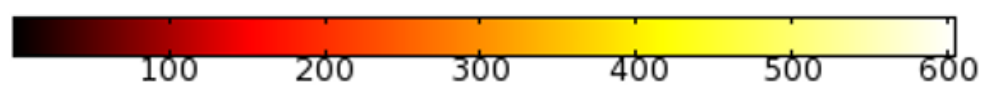

d.

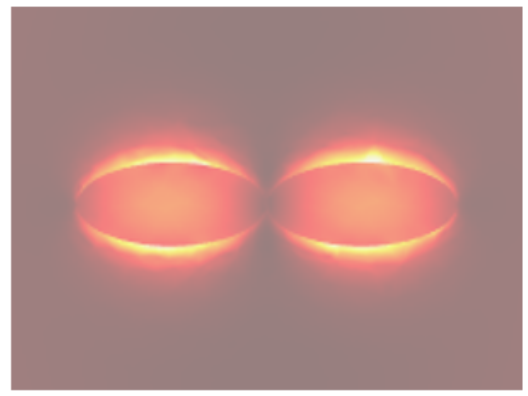

$\Delta 550$

\section{Figure 4}

a. Truncated Gold nano-elliptical dimer (NED) with gain media coating and separation $\mathrm{S}=5 \mathrm{~nm} \mathrm{~b}$. ECS of NED for $x$-y luminance c. NFE of NED for $x$-polarization d. NFE of NED for y-polarization 

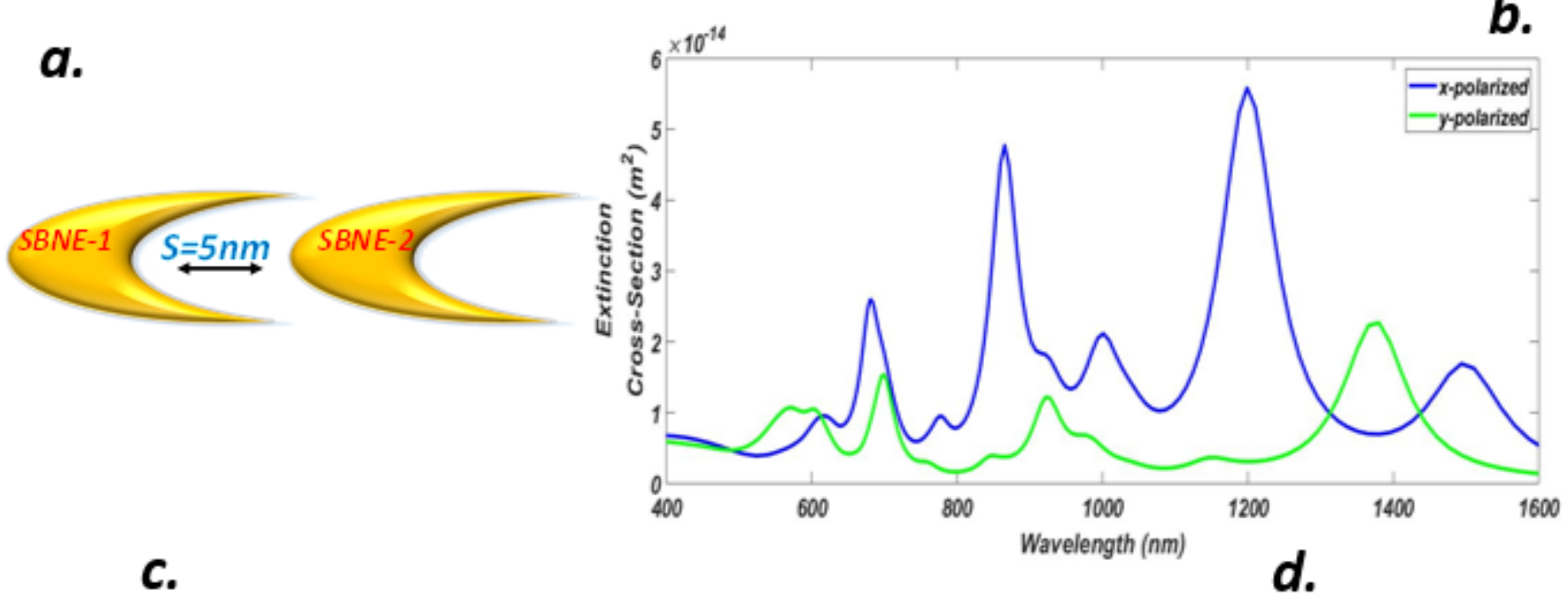

\section{c.}
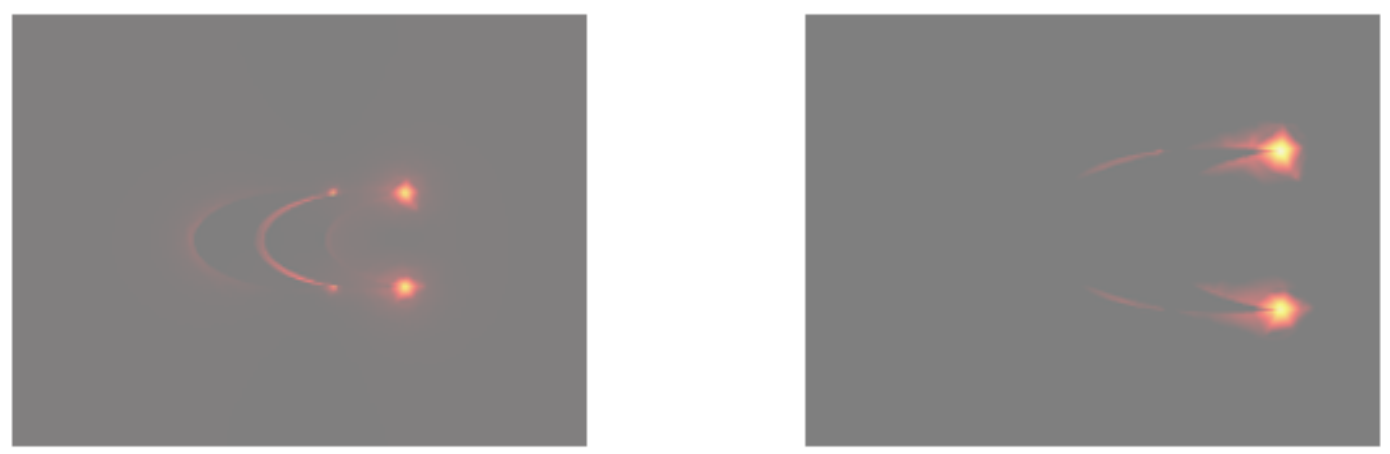

20

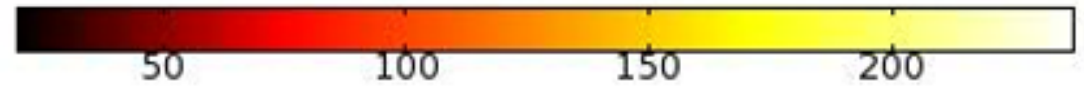

$\Delta 220$

\section{Figure 5}

a. Truncated Gold symmetry broken nano-elliptical dimer (SBNED) with gain media coating and separation $S=5 \mathrm{~nm} b$. ECS of SBNED for $x$-y luminance $c$. NFE of SBNED for $x$-polarization d. NFE of SBNED for y-polarization 


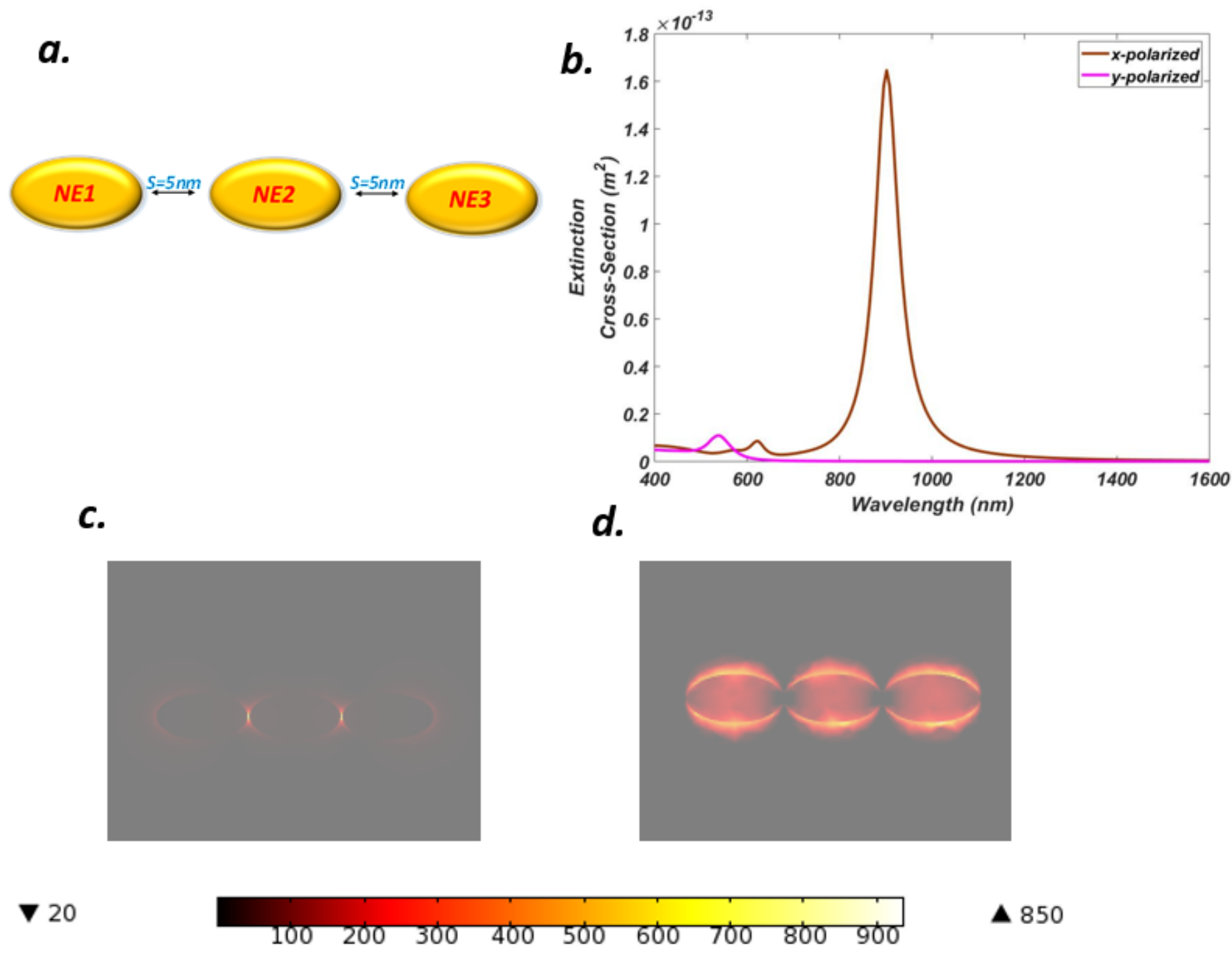

Figure 6

a. Truncated Gold linear chain nano-elliptica trimer (LCNET) with gain media coating and separation $S=5$ $\mathrm{nm}$ b. ECS of LCNET for $x-y$ luminance $c$. NFE of LCNET for $x$-polarization d. NFE) of LCNET for $y$ polarization. 

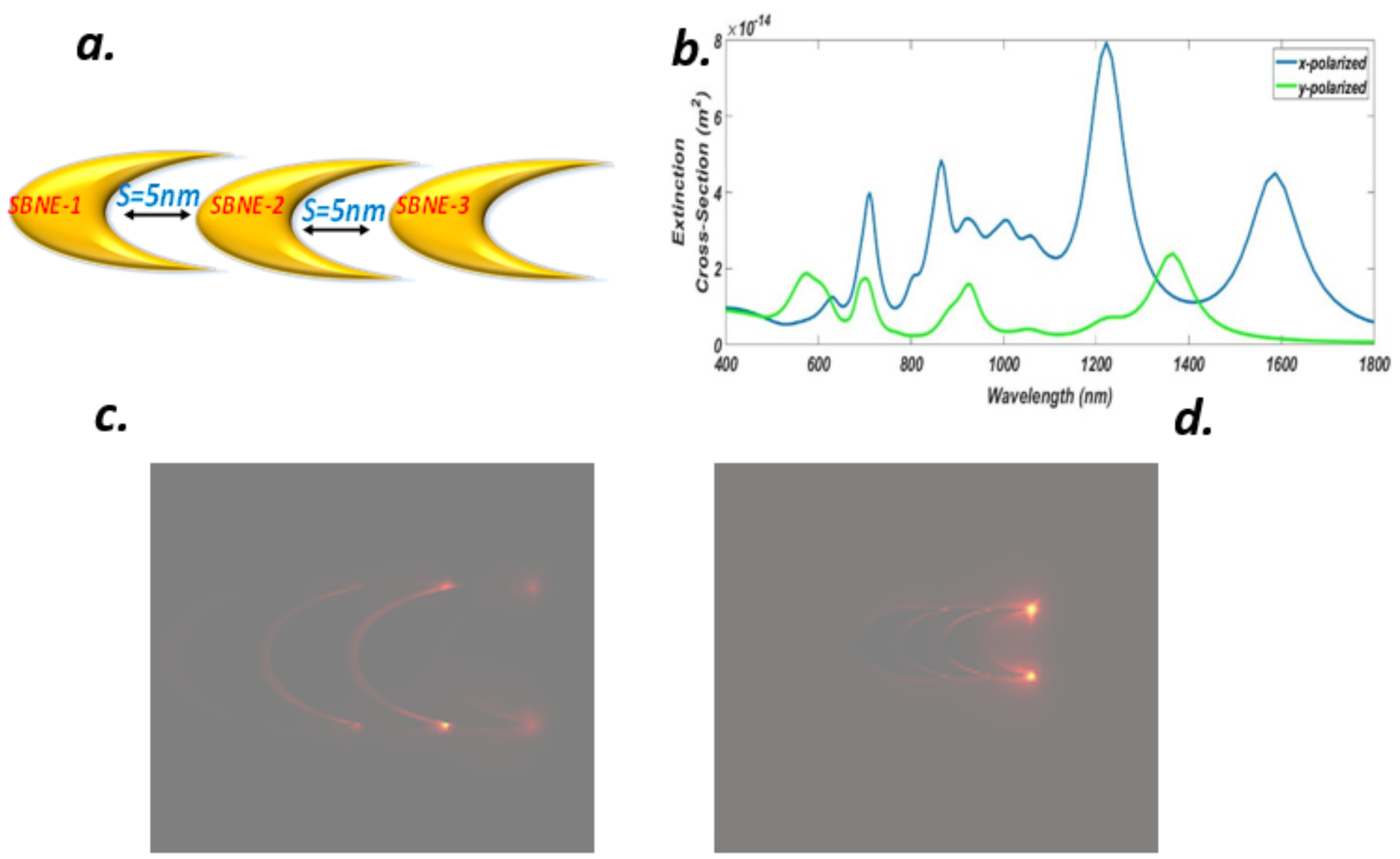

$\boldsymbol{\nabla} 100$

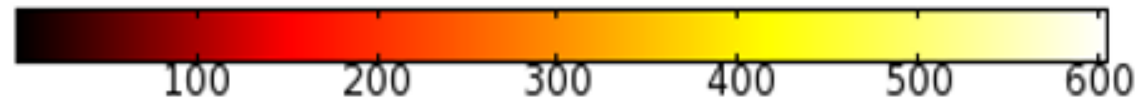

$\Delta 550$

\section{Figure 7}

a. Truncated Gold symmetry broken linear chain nano-elliptica trimer (SBLCNET) with gain media coating and separation $S=5 \mathrm{~nm}$ b. ECS of SBLCNET for $x$-y luminance c. NFE of SBLCNET for x-polarization $d$. NFE of SBLCNET for y-polarization 
$\boldsymbol{a}$
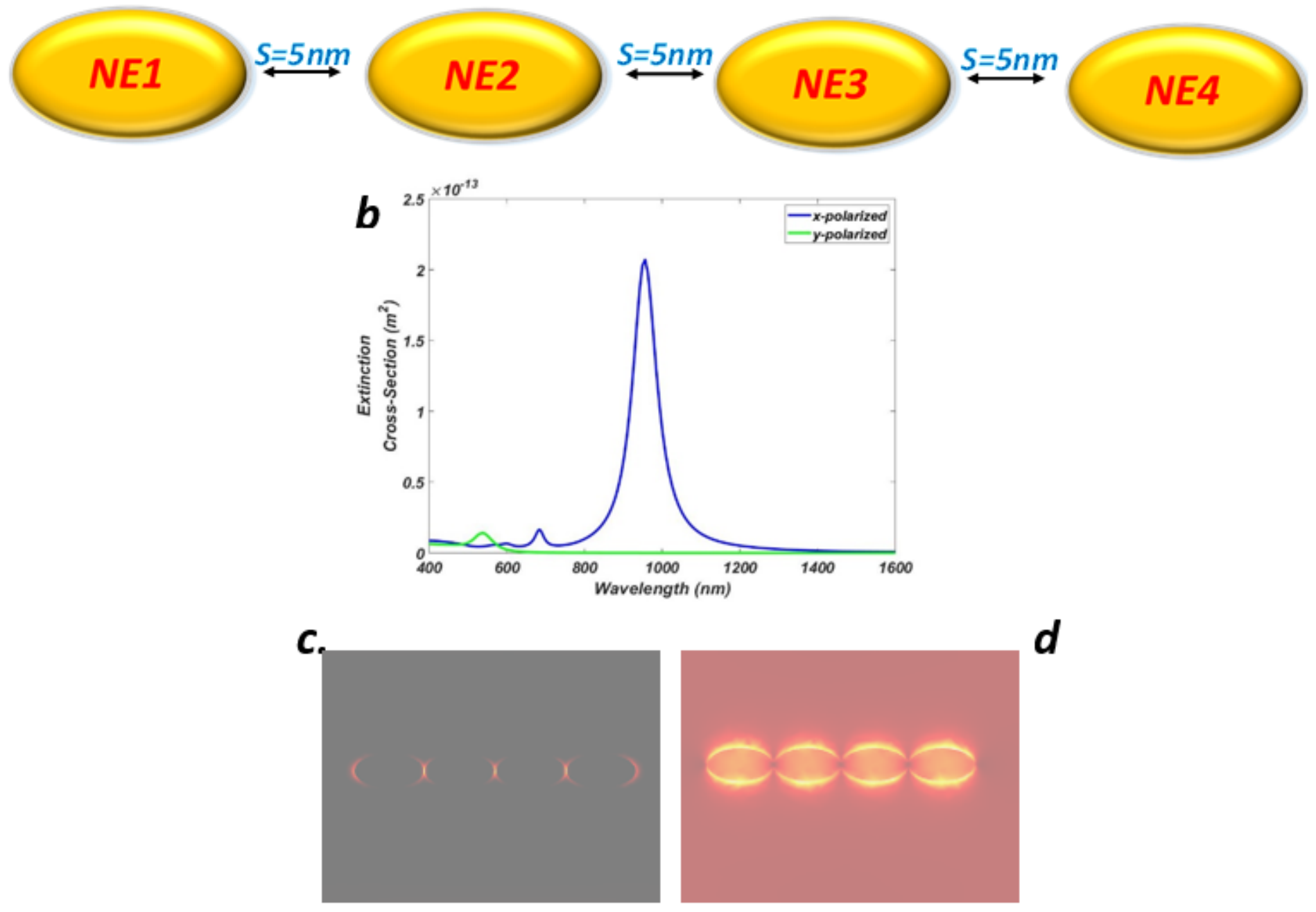

$\mathbf{\nabla} 20$

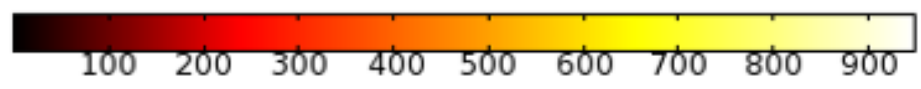

$\Delta 950$

Figure 8

a. Truncated Gold linear chain nano-elliptical quadramer (LCNEQ) with gain media coating and separation $\mathrm{S}=5 \mathrm{~nm}$ b. ECS of LCNEQ for $x$-y luminance $c$. NFE of LCNEQ for $x$-polarization d. NFE of LCNEQ for y-polarization. 
a.
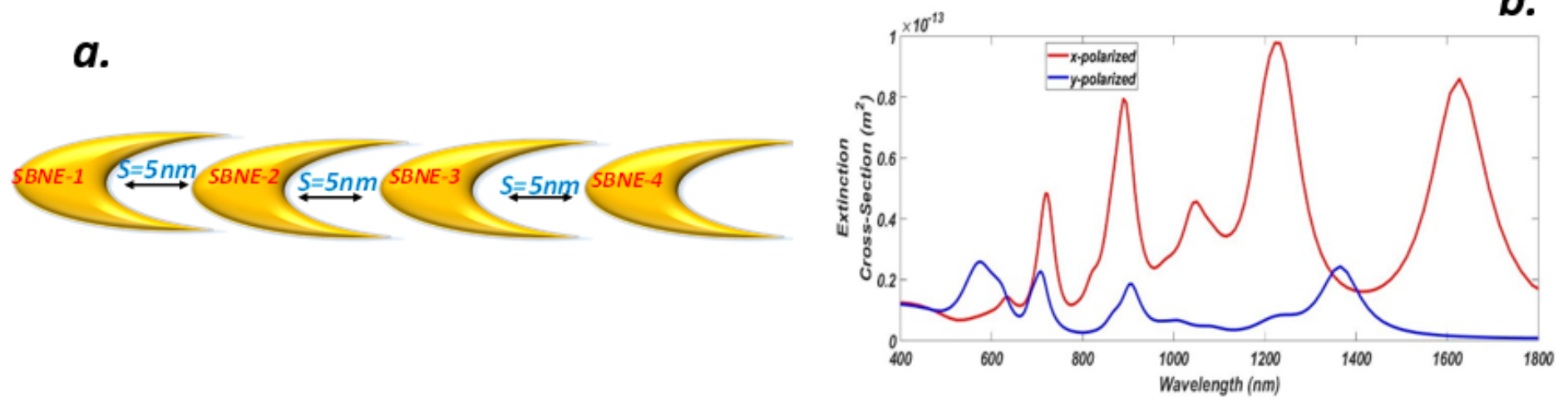

b.

C.
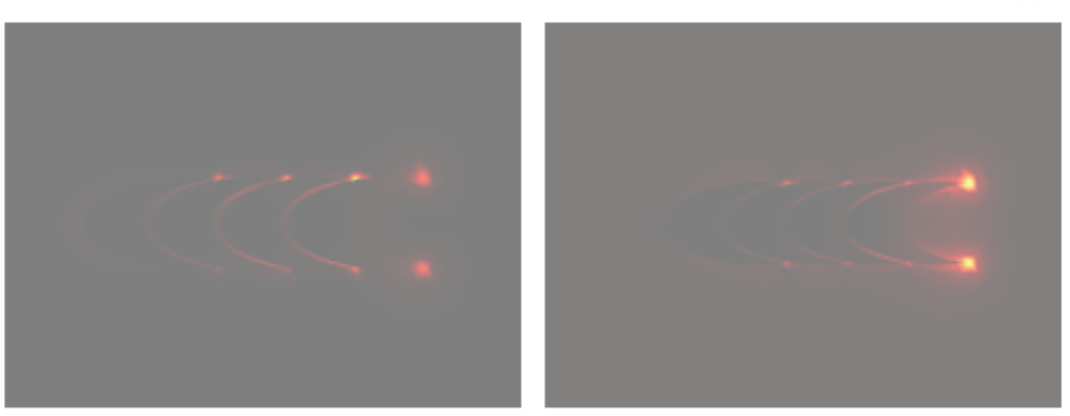

d.

$\mathbf{\nabla} 100$

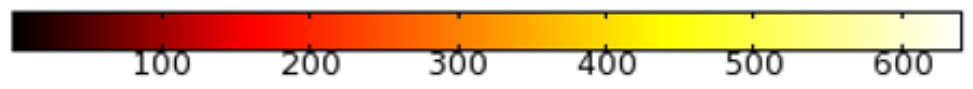

$\boldsymbol{\Delta} 550$

\section{Figure 9}

a. Truncated Gold symmetry broken linear chain nano-elliptical quadramer (SBLCNEQ) with gain media coating and separation $S=5 \mathrm{~nm}$ b. ECS of SBLCNEQ for $x-y$ luminance c. NFE of SBLCNEQ for $x-$ polarization d. NFE of SBLCNEQ for y-polarization. 


\section{x-polarization luminance}

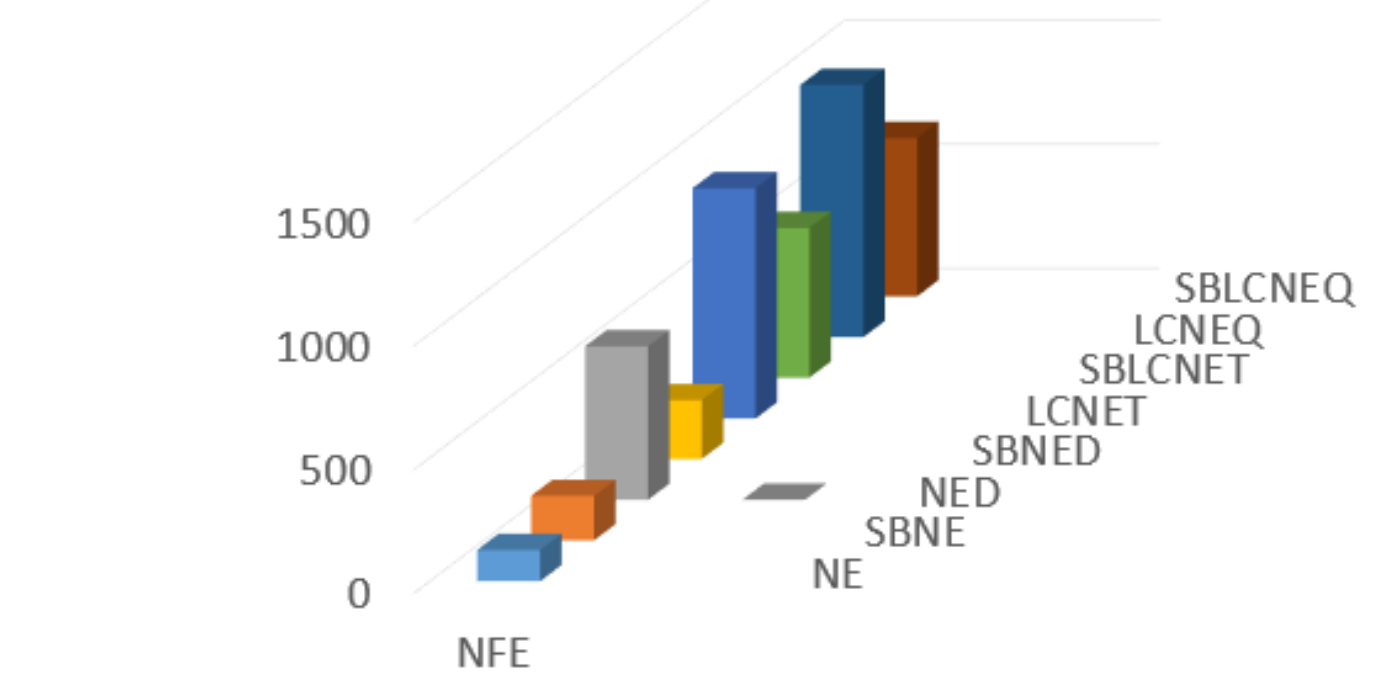

$\square \mathrm{NE} \quad$ SBNE $\square$ NED $\square$ SBNED $\quad$ LCNET $\quad$ SBLCNET $\square$ LCNEQ $\square$ SBLCNEQ

\section{Figure 10}

Near Field Enhancement (NFE) of normal and symmetry broken nano-elliptical structures for xpolarization luminance 


\section{y-polarization luminance}

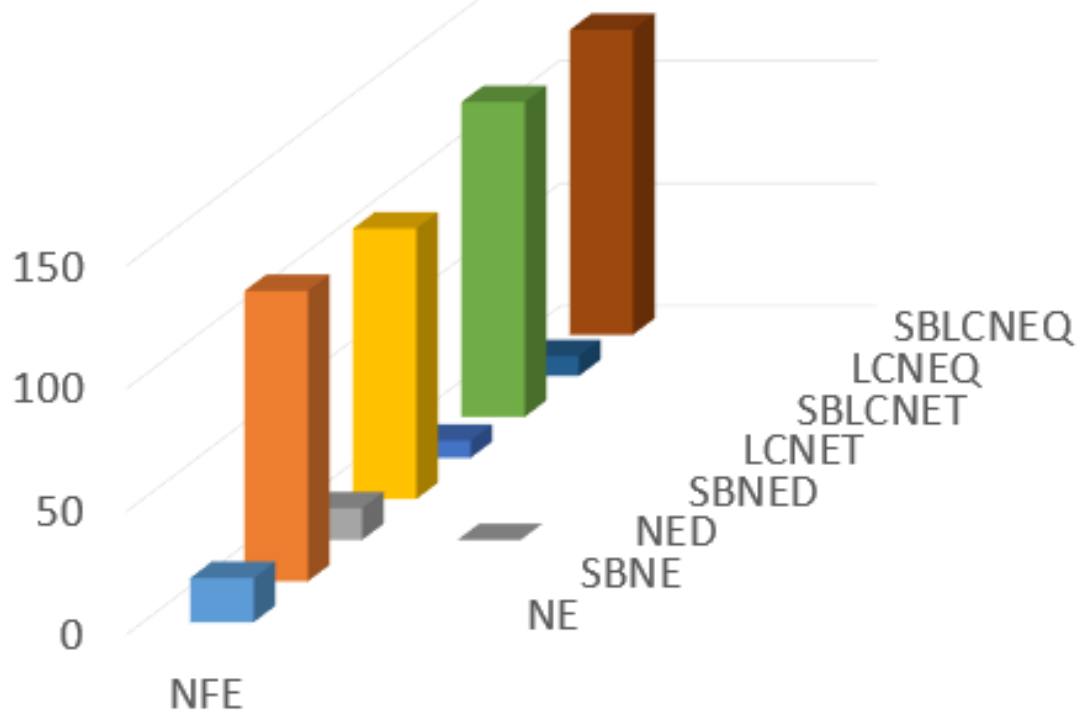

$\square \mathrm{NE} \quad$ SBNE $\square$ NED $\square$ SBNED $\square$ LCNET $\square$ SBLCNET $\square$ LCNEQ $\square$ SBLCNEQ

\section{Figure 11}

Near Field Enhancement (NFE) of normal and symmetry broken nano-elliptical structures for $y$ polarization luminance 